\title{
Chapter 79 \\ Bladder Cancer Survivorship and Patient \\ Reported Outcome Measures-Code 5 \\ Impact of Operation on General Health
}

There were 7 patients involved with TURBTs.

\begin{tabular}{|l|l|}
\hline $\begin{array}{l}\text { Table Code (5) Impact of operation on gen- } \\
\text { eral health }\end{array}$ & Comments made were as followed \\
\hline Impact of operation & No side effects \\
\hline Impact of operation & $\begin{array}{l}\text { ED afterwards-1 month-age dependant- } \\
\text { would initially try to see if it resolved, then } \\
\text { seek CNS help—an opt in pathway would } \\
\text { help }\end{array}$ \\
\hline Impact of operation & $\begin{array}{l}\text { Recurrent problems with chest and uti-man- } \\
\text { aged medically—really happy }\end{array}$ \\
\hline
\end{tabular}

There was no impact of procedure experience for BCG/MMC patients.

There were 10 patients who underwent radical cystectomy. Comments made were as follows:

\begin{tabular}{|l|l|}
\hline $\begin{array}{l}\text { Table Code (5) Impact of operation on gen- } \\
\text { eral health }\end{array}$ & Comments made were as followed \\
\hline Requirement for pathway & $\begin{array}{l}\text { Peer support group with CNS beforehand } \\
\text { would have helped }\end{array}$ \\
\hline Nutritionist requirement & $\begin{array}{l}\text { Nutritionist needed. Taken erections away, but } \\
\text { age related }\end{array}$ \\
\cline { 2 - 2 } & $\begin{array}{l}\text { Lymphoedema-currently present. Pathway } \\
\text { needed with CNS, but difficult to get a time } \\
\text { as to when to start-pt dependant }\end{array}$ \\
\hline Impact of operation & Problem post op-hernia post op \\
\hline
\end{tabular}

S. S. Goonewardene et al., Management of Non-Muscle Invasive Bladder Cancer, https://doi.org/10.1007/978-3-030-28646-0_79 